\title{
Numerical Study on Bubble Dynamic by Pulsed Electric Discharge in Water
}

\author{
Zhibo Yang ${ }^{1}$ and Chaohai Zhang ${ }^{2,3}$ \\ 1. School of Electrical Engineering, Northeast Dianli University, Jilin 132012, China \\ 2. School of Electrical Engineering, Harbin Institute of Technology, Harbin 150001, China \\ 3. The State Grid Electric Power Research Institute, Wuhan 430074, China
}

\begin{abstract}
The bubble dynamics by pulsed electric discharge in water at or near a solid surface is numerically calculated. In this model, the Navier-Stokes equations are solved accurately and efficiently. The bubble-water interface is tracked by the VOF (volume of fluid) method. Based on the experimental condition that the bubble attaches to the solid surface, the stand-off parameter, $\gamma=0.8$, has been used to simulate the bubble profile in the growth and collapse phase. Then, the jet velocity on the solid surface has been calculated, which can generate a high localized pressure with high magnitude of $2.11 \times 10^{8} \mathrm{~Pa}$, with short duration of $0.1 \mathrm{~s}$. Our numerical model is first validated against experimental data from the literature on bubble dynamics. Good agreement was obtained between experimental data and numerical simulation results.
\end{abstract}

Key words: Pulsed electric discharges in water, bubble dynamics, pulsation, water jet, water hammer pressure, Navier-Stokes equation.

\section{Introduction}

PEDW (pulsed electric discharges in water) has recently attracted significant interest for their applications, such as high repetition rate switch [1], waste water treatment [2], under water sound source [3]. The streamer by PEDW interacts with fluid of water to initiate physical and chemical process, including a strong electric field, intense UV radiation, shockwave, bubble, and formation of various chemically active species.

Many studies have used pulsed laser source to generate laser-induced bubble in water by high speed camera. The laser-induced bubble can be created at a chosen distance from the solid surface, and bubble profile is similar with laser plasma channel [4]. However, the streamer-induced bubble attaches to the electrode tip, and expands by gaseous filaments collection. Akiyama et al. [5] have presented experimental studies on streamer-induced bubble.

Corresponding author: Zhibo Yang, Ph.D., research fields: underwater discharge and its application.
As the process of jet formation and distortion of bubble-water interface near a solid surface is extremely complicated. Numerical simulation is a powerful way to gain an understanding of the growth and collapse mechanism. Stephane et al. [6], Minsier [7], and Brujan [8] have presented several models on bubble dynamic process. Some of these models considered the water jet penetration in the last collapse phase. However, for the streamer-induced bubble, it is difficult to simulate the bubble dynamic process, because the initial radius of bubble is unknown.

In this paper, a numerical method has been developed to simulate the dynamics of a single streamer-induced bubble including water jet penetration process. The stand-off parameter $\gamma$ was a choice based on the experimental data of Ref. [5]. The jet velocity impact on the solid surface has been calculated, which can generate a high localized pressure with short duration. 


\section{Numerical Method}

In both the growth phase and collapse phase of a bubble, the flow is assumed to be laminar, bubble is compressible and assumed to be an ideal gas without considering mass transfer and the flow without swirl, the water surrounding the bubble is assumed incompressible. The VOF method is used to track the interface between bubble and water.

\subsection{Navier-Stokes Equations}

The Navier-Stokes equations express mass, momentum, and energy balance, respectively, for the entire flow field:

The continuity equation is:

$$
\frac{\partial \rho}{\partial t}+\nabla \cdot(\rho \vec{v})=0
$$

where $\rho$ is the fluid density and $\vec{v}$ is the velocity field in the whole domain.

The momentum equation is:

$$
\begin{gathered}
\frac{\partial}{\partial t}(\rho \vec{v})+\nabla \cdot(\rho \vec{v} \vec{v})= \\
-\nabla P_{f}+\nabla \cdot\left[\mu\left(\nabla \vec{v}+\nabla \vec{v}^{T}\right)\right]+\rho \vec{g}+\vec{F}_{S}
\end{gathered}
$$

where $P_{f}$ is the pressure domain, $\vec{g}$ is the gravity force, and $\vec{F}_{S}$ is the surface tension force, which is calculated as $\vec{F}_{S}=\sigma \kappa \tilde{m}$, where $\kappa$ is the curvature and $\tilde{m}$ is the normal vector to the interface.

The energy equation is:

$$
\frac{\partial}{\partial t}\left(\rho E_{f}\right)+\nabla \cdot\left(\vec{v}\left(\rho E_{f}+P_{f}\right)\right)=\nabla \cdot\left(k_{e f f} \nabla T_{f}\right)
$$

where $E_{f}$ is the enthalpy of the fluid, $T_{f}$ is the temperature field, and $k_{\text {eff }}$ is the conductivity coefficient of the fluid.

The surface tension term $\vec{F}_{S}$ in Eq. (2) is computed using the continuum surface force model proposed by Brackbill et al. [9]. In this model, the surface tension at the bubble-water interface is expressed as a volume force in the momentum equation using the divergence theorem. This volume force is given by,

$$
\vec{F}_{S}=\sigma \frac{\rho \kappa \nabla \alpha_{L}}{0.5\left(\rho_{L}+\rho_{g}\right)}
$$

where $\sigma$ is the surface tension, $\kappa$ is the curvature of the interface, and $\alpha_{L}$ is the volume fraction of the water. The curvature is defined as $\kappa=\nabla \cdot\left(\frac{n}{\|n\|}\right)$, where $n$ is the surface normal defined as a function of the gradient of $\alpha_{L}$.

\subsection{VOF Method}

As the VOF method is based on a one fluid approach, the fluid density is calculated according to the following equation:

$$
\rho=\rho_{l} F+\rho_{g}(1-F)
$$

where $F$ is the volume fraction of liquid.

In our VOF method, the method of tracing inter-phase boundary is achieved by solving volume fraction continuity equation of one phase or several phases. As for the $q$ phase, volume fraction equation is

$$
\frac{\partial F_{q}}{\partial t}+\vec{v}_{q} \cdot \nabla F_{q}=0
$$

Equation is not solving the primary phase, primary phase volume fraction calculation is based on the following constraints:

$$
\sum_{q=1}^{2} F_{q}=1
$$

\subsection{Initial Conditions}

It should be noted that simulation of very first stage of bubble growth is beyond the scope of the present work. The explanation has been stated in Section 3.1. Our bubble dynamics simulations start from an initial pre-existing spherical bubble with radius $R_{0}$. The bubble is assumed initially at rest because the residuals never converged when a velocity field is initially applied. A pressure discontinuity is initially applied at the bubble-water interface. The pressure is $1.01 \times 10^{5}$ $\mathrm{Pa}$ in ambient water and $P_{0}$ inside the bubble. If a temperature discontinuity was applied at bubble-water 
interface, results would be wrong and mesh dependent. Therefore, based on the work of Nagrath et al. [10], the initial temperature field $T_{0}$ as a function of the distance $d$ from the solid surface to initial bubble center is expressed as a normal distribution,

$$
T_{0}(s)=T_{a m b}+T_{c 0} e^{-1 / 2\left(2000 d / R_{0}\right)^{2}}
$$

where $T_{a m b}=300 \mathrm{~K}$ is the ambient temperature in the water, $T_{c 0}$ is the difference between the temperature at center of the bubble and the ambient temperature.

The choice of the initial conditions, including the initial bubble radius $R_{0}$, initial pressure inside bubble $P_{0}$, and $T_{c 0}$, is nontrivial by the lack of experimental data in the early stages of streamer-induced bubble generation. Therefore, the initial conditions have been determined such that the bubble reaches a maximum radius in agreement with available experimental data.

Our initial bubble is placed in liquid water at a fixed distance from a solid surface. It should be considered the stand-off parameter $\gamma$, which is defined as the ratio between the distance from the bubble center to the solid surface and the maximum bubble radius. $\gamma=s / R_{\max }$, $s$ is the distance between the bubble center and the solid surface of electrode. Our initial bubble radius $R_{0}$ has been measured by the value of $4 \mathrm{~mm}$ from stable growth phase at $182.2 \mu$ s (the choice of initial bubble radius has also been stated in Section 3.1), thus $s$ should be set to $4 \mathrm{~mm}$. The maximum radius has been measured by the value of $5 \mathrm{~mm}$ at $442.2 \mu \mathrm{s}$. Therefore, the stand-off parameter $\gamma$ could be calculated by the value of 0.8 . Then, it could be calculated that, $P_{0}=2.8 \times 10^{8} \mathrm{~Pa}$, and $T_{c 0}=2000 \mathrm{~K}$ for $\gamma=0.8 \mathrm{in}$ the growth phase. In the collapse phase, the computational domain is set from the largest bubble radius initially till impact to the surface of electrode. The velocity is set to zero to initialize the flow in the whole domain. The pressure inside the bubble, $P_{R}=400 P a$ for $\gamma=0.8$, ambient water pressure, $P_{\infty}=1.01 \times 10^{5} \mathrm{~Pa}$. In the whole simulation, the buoyancy and gravity are neglected due to short interaction time. The surface tension is set to default value of $0.073 \mathrm{n} / \mathrm{m}$. Fig. 1 shows the initial condition of bubble in the growth phase.

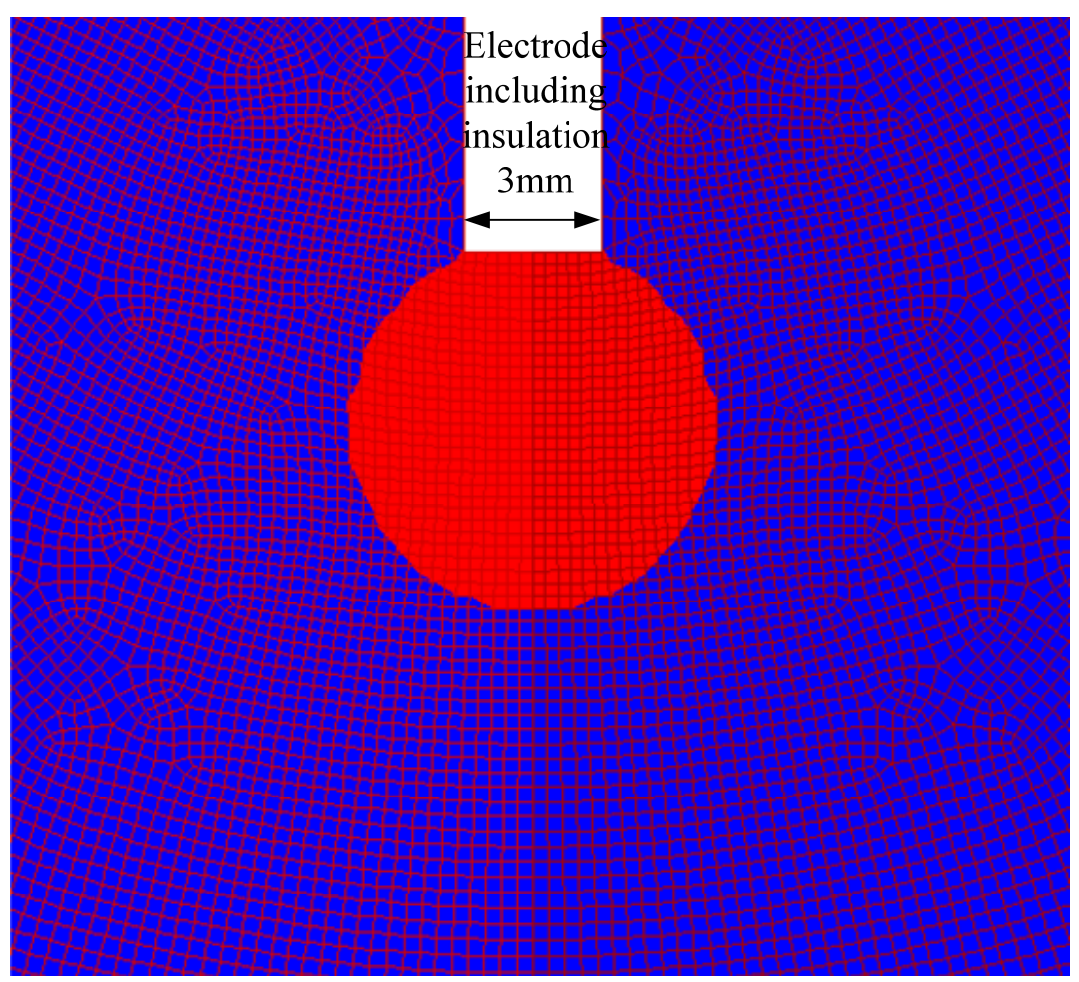

Fig. 1 Meshes of bubble profile at initial radius of $4 \mathrm{~mm}$, for $\gamma=0.8$. 


\section{Results and Discussion}

\subsection{Experimental Data Explanation}

In the growth phase of a streamer-induced bubble, as shown in Fig. 10 of Ref. [5], where (a)-(f) are taken at $2.2 \mu \mathrm{s}, 22.2 \mu \mathrm{s}, 82.2 \mu \mathrm{s}, 182.2 \mu \mathrm{s}, 320.2 \mu \mathrm{s}$, and 442.2 $\mu \mathrm{s}$, respectively. It indicates two aspects: one is the coalescence of gaseous filaments to a main bubble in the growth phase. The streamer channels propagate uniformly under water, and the internal energy of gaseous filaments is in high magnitude after streamer channels faded. As shown in Fig. 10 (a)-(d) of Ref. [5], the gaseous filaments become shorter and thicker, and merge into the main bubble till $182.2 \mu$ s. Therefore, till 182.2 $\mu \mathrm{s}$, the main bubble is in the unstable growth phase, and it is difficult to consider the surface tension act to the main bubble due to high pressure inside the gaseous filaments. The other aspect is that the main bubble expands, and heats by electrode tip during the whole growth phase. As shown in Fig. 10 (d)-(f) of Ref. [5], the main bubble remains generally with expansion in almost spherical shape. It could be believed that the gaseous filaments insulate from the main bubble, and shrink starting from $182.2 \mu \mathrm{s}$. Therefore, from $182.2 \mu \mathrm{s}$, the main bubble is heated by the electrode tip in the stable growth phase, acted by surface tension.

The bubble reaches the maximum size when the velocity of bubble-water interface is zero. Then, the bubble begins to collapse. Fig. 11 of Ref. [5] shows the collapse of bubbles, where (a)-(c) are taken at 500, 680, and $740 \mu \mathrm{s}$, respectively. A satellite bubble (gaseous filament) exists near the main bubble, which insulates from the main bubble in vibration state. This phenomena can be contributed the need for internal energy promote the dynamics of gaseous region [11].

\subsection{Numerical Simulation Results}

Fig. 2 shows the bubble profile at the growth phase of 182 s, 262 s, 402 s, and 442 s, respectively. The bubble keeps spherical profile during increasing size. It reaches the maximum radius of $5 \mathrm{~mm}$ at $442 \mathrm{~s}$.

Fig. 3 (a)-(b) shows bubble profiles during early collapse phase at $500 \mathrm{~s}$ and $680 \mathrm{~s}$ respectively. In the early collapse phase, the bubble size decreases. The bubble is seen to move toward the solid surface. The bottom of bubble-water interface became flattened. The results are in a good agreement with Fig. 11 (a)-(b) of Ref. [5], and collapse times are on the similar order. Fig. 3 (c)-(d) shows jet penetration inside the bubble during the last collapse phase. Jet formation starts when the bottom of the bubble-water interface had an ordinate smaller than any other points of the bubble-water interface, as shown in Fig. 3 (c). At $\mathrm{t}=740 \mathrm{~s}$, the water jet reaches the top bubble-water interface, and water jet impacts on the solid surface finally.

Fig. 4 compares the radius of bubble in the growth and collapse phase, including experimental data and simulation results. It indicates that the simulation results are good agreement with experimental data. As shown in Fig. 4, the data of blue solid circle indicate time variation of the main bubble radius in the unstable growth phase; the data of red solid circle indicate time variation of bubble radius in the stable growth phase, besides, of bubble radius in early collapse phase; the data of green solid circle indicate time variation of bubble radius in the last collapse phase, besides, of bubble radius in the re-expand phase.

\subsection{Jet Velocity}

Fig. 5 shows the jet velocity field at the moment of impact at $740 \mu \mathrm{s}$. The velocity normal to the solid surface is a main factor as impact velocity for the red arrow. The impact velocity reaches the maximum value of $147 \mathrm{~m} / \mathrm{s}$, when the flow impact to the solid surface is at $740 \mu \mathrm{s}$. There are also eddy flows with the range from 47 to $95 \mathrm{~m} / \mathrm{s}$. The jet velocity generation is due to unbalance of momentum acted on between the top of bubble-water interface (attaching to electrode tip) and bottom of bubble-water interface. 

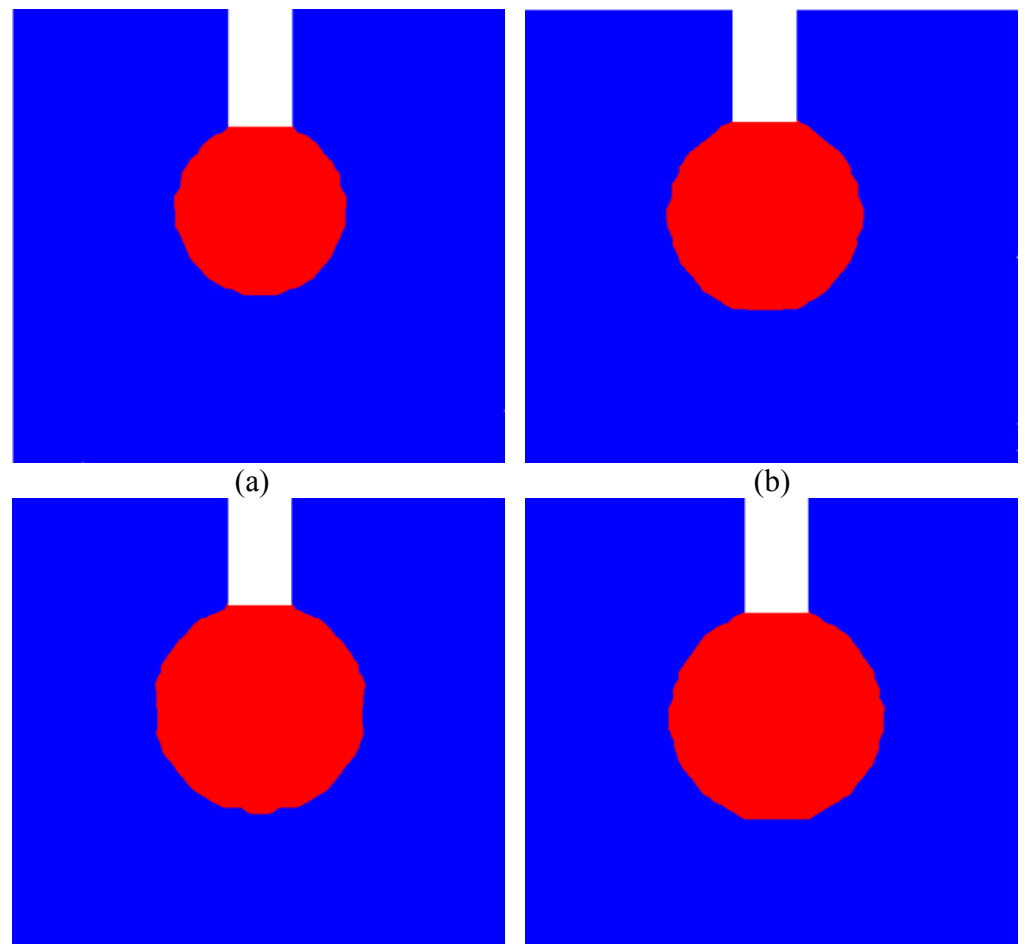

(b)

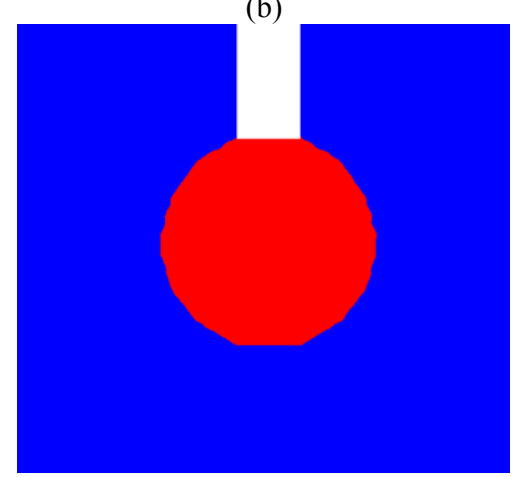

(c)

(d)

Fig. 2 Bubble profile in the growth phase, for $\gamma=0.8$, where (a)-(f) are taken at $182 \mathrm{~s}, 262 \mathrm{~s}, 402 \mathrm{~s}$, and $442 \mathrm{~s}$, respectively.

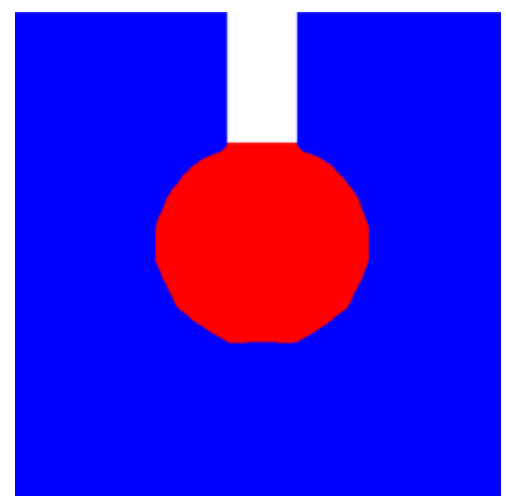

(a)

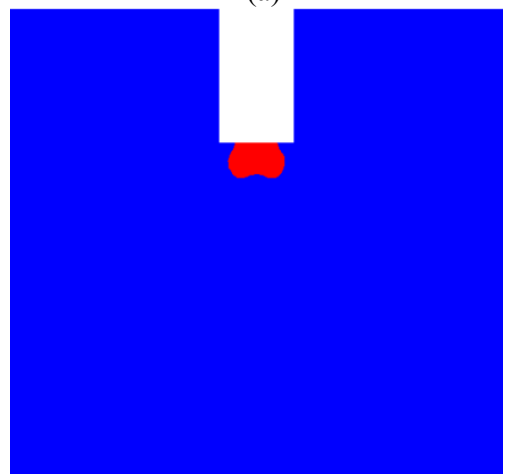

(c)

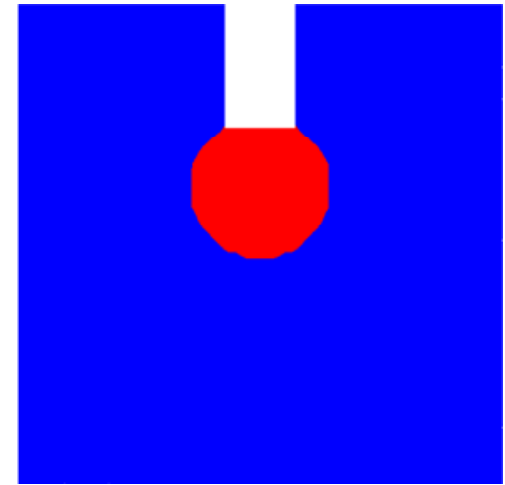

(b)

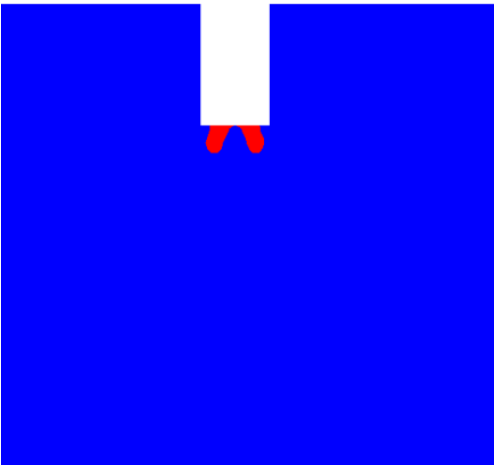

(d)

Fig. 3 Bubble collapse phase, where (a)-(b) were taken at $500 \mathrm{~s}$ and $680 \mathrm{~s}$, (c)-(d) were taken at $734 \mathrm{~s}$ and $740 \mathrm{~s}$. 


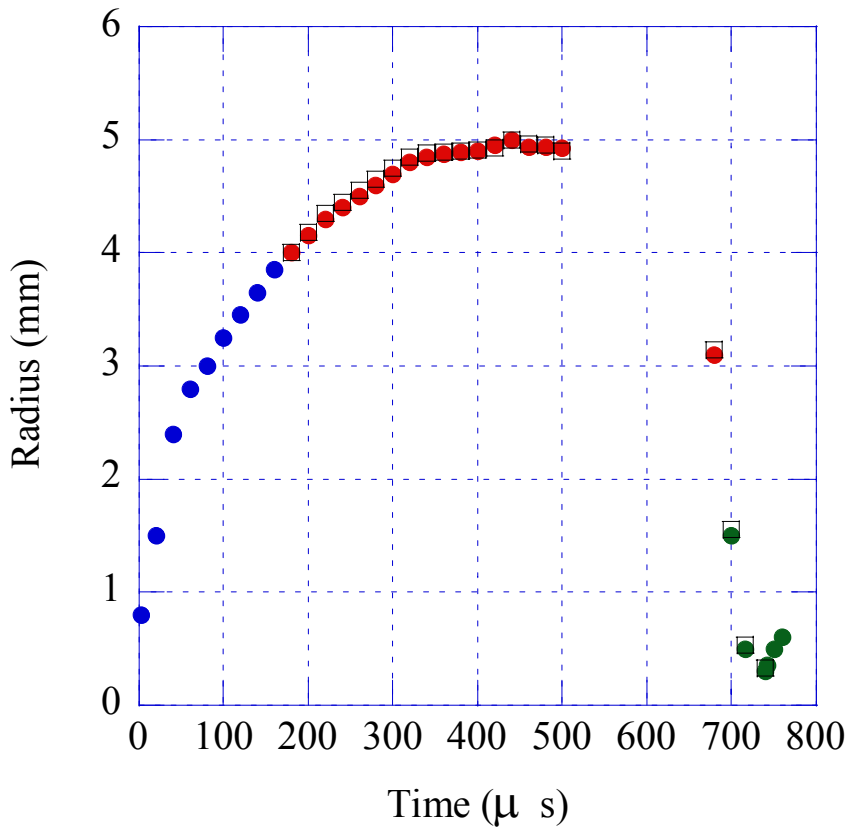

Fig. 4 Time variation of bubble radius for experimental data (the solid circle) and simulation data (the hollow square). After $740 \mu \mathrm{s}$, the bubble begins to re-expand and take the second oscillated process.

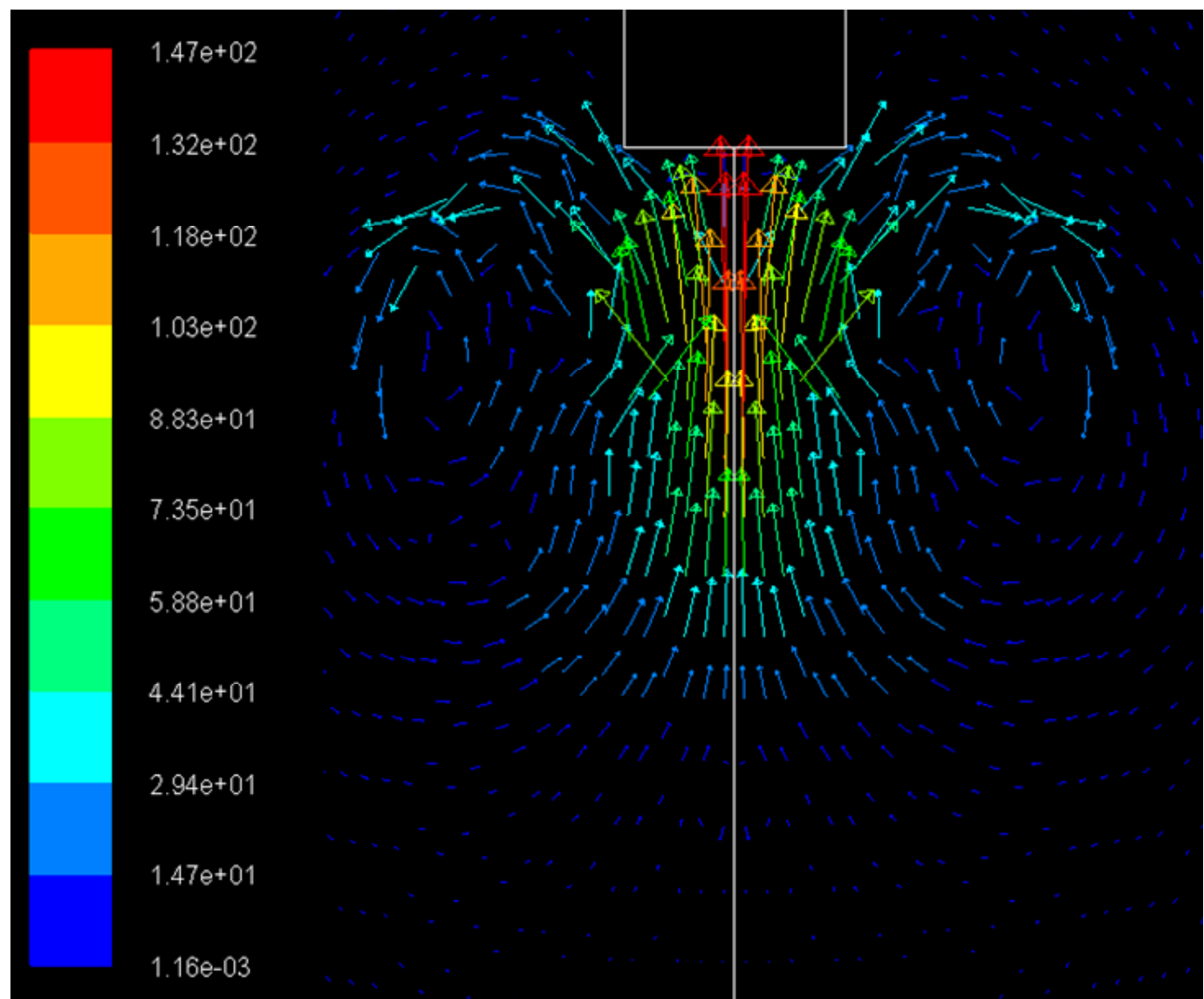

Fig. 5 The jet velocity field at the moment of impact at $740 \mu \mathrm{s}$. 
Table 1 Physics properties in experimental condition.

\begin{tabular}{llll}
\hline$\rho_{L}(\mathrm{~kg} / \mathrm{m} 3)$ & $c_{L}(\mathrm{~m} / \mathrm{s})$ & $\rho_{S}\left(\mathrm{~kg} / \mathrm{m}^{3}\right)$ & $c_{S}(\mathrm{~m} / \mathrm{s})$ \\
\hline $1.0 \times 10^{3}$ & $1.5 \times 10^{3}$ & $9.0 \times 10^{3}$ & $3.8 \times 10^{3}$ \\
\hline
\end{tabular}

\subsection{Damage Capability of Jet}

The impact of a water jet on a solid wall generates an initial water hammer pressure, is given by Ref. [12].

$$
P_{W H}=\frac{\rho_{L} c_{L} \rho_{S} c_{S}}{\rho_{L} c_{L}+\rho_{S} c_{S}} v_{i m p}
$$

where $\rho_{L}$ and $c_{L}$ are density and sound velocity of the jet medium respectively, $\rho_{S}$ and $c_{S}$ are corresponding values for the solid wall. $v_{i m p}$ is the velocity impact to the solid surface. According to experimental data, water and copper are selected as materials respectively. Table 1 shows the physics properties in experimental condition. $v_{i m p}$ is the maximum value of $147 \mathrm{~m} / \mathrm{s}$. It can be calculated that the water hammer pressure is $2.11 \times 10^{8} \mathrm{~Pa}$.

On the other hand, the duration of interaction also determines the resulting damage. The duration of interaction is determined by the time the rarefaction wave starting at the moment of impact from border of the jet expand to the jet center [12]. As shown in Fig. 12 (a) of Ref. [5], the mean radius of water jet is measured as low as $150 \mu \mathrm{m}$, the water hammer pressure acts for a time $\tau_{w}=R_{j e t} / c_{L} \approx 0.1 \mu s \quad\left(R_{j e t}\right.$ is the radius of the jet). After this time, the pressure decreases to the stagnation pressure. Therefore, in the last collapse phase, water jet can generate the high-pressure region on the solid surface. Water hammer pressure is a violent pulse with high magnitude and short duration, which can damage the surface of electrode.

Immediately after the jet impact, the compressible nature of water becomes dominant over a very short interval, however, the numerical simulation based on the incompressible theory cannot capture this phenomenon. The jet flow through the bubble (at this time it would be still called bubble, although bubble-water interface has been distorted) can change the topology of the cavity and the bubble profile becomes inverted funnel shape, as shown in Fig. 12 (a) of Ref. [11]. The bubble remains to re-expand from a minimum size, and a shock wave generates in following event, but the present numerical simulation cannot allow the motion to be tracked further in time.

\section{Conclusions}

In this paper, we numerically studied the streamer-induced bubble dynamics including jet penetration near the solid surface of electrode. The simulation results provided numerical method to study cavitation bubble and water jet, compared to experimental data. The following conclusions are inferred:

(1) The evolution of bubble profile with time was good agreement with experimentally observed bubble dynamics for $\gamma=0.8$.

(2) In the early collapse phase, the bubble started to shrink, moved to the solid surface, and bottom of bubble-water interface became flatten along the solid surface; in the last collapse phase, jet formation started from the bottom of bubble-water interface, then reached the top interface, with a high velocity impact. The jet velocity generation is caused by the unbalance of momentum acted on between the top and bottom of bubble-water interface.

(3) Water jet can generate the high-pressure region on the solid surface. Based on the experimental condition, water hammer pressure was a violent pulse with high magnitude of $2.11 \times 10^{8} \mathrm{~Pa}$, with short duration of $0.1 \mathrm{~s}$.

\section{References}

[1] Xiao, S., Kolb, J., Kono, S., Katsuki, S., Laroussi, M., and Schoenbach, K. 2004. "High Power Water Switches: Postbreakdown Phenomena and Dielectric Recovery." IEEE Tran. Dielectr. Electr. Insul. 11 (4): 604-12.

[2] Yano, T., Shimomura, N., Uchiyama, I., Fukawa, F., Teranishi, K., and Akiyama, H. 2009. "Decolorization of Indigo Carmine Solution Using Nanosecond Pulsed Power." IEEE Tran. Dielectr. Electr.Insul. 16 (4): 1081-7. 
[3] Yan, P., Sun, Y. H., Zhou, Y. X., and Jin, M. J. 2004. "Underwater Sound Source Based on Pulsed Corona Discharge." Annual Report Conference on Electrical Insulation and Dielectric Phenomena.

[4] Johnsen, E. and Colonius, T. 2011. "Shock-Induced Collapse of a Gas Bubble in Shockwave Lithotripsy." Journal of the Acoustical Society of America 124: 2011-20.

[5] Akiyama, M., Yang, Z. B., Sebastian, G., Hosseini, S. H. R., and Akiyama, H. 2014. "Observation of Underwater Streamer Discharges Produced by Pulsed Power Using High Speed Camera." IEEE Transactions on Plasma Science 42: 3215-20.

[6] Stephane, P., and Stephane, Z. 2002. "Bubble Collapse near a Solid Boundary: A Numerical Study of the Influence of Viscosity." Journal of Fluid Mechanics 464: 137-63.

[7] Minsier, V., Wilde, J. D., and Proost, J. 2009. "Simulation of the Effect of Viscosity on Jet Penetration into a Single Cavitating Bubble." Journal of Applied Physics 106:
0849061-10.

[8] Brujan, E. A., Nathen, K., Schmidt, P., and Vogel, A. 2001. "Dynamics of Laser-Induced Cavitation Bubble near Elastic Boundaries: Influence of the Elastic Modulus." Journal of Fluid Mechnaism 433: 283-314.

[9] Brackbill, J. U., Kothe, D. B., and Zemach, C. 1992. "A Continuum Method for Modeling Surface Tension." Journal of Computer Physics 100: 335-41.

[10] Nagrath, S., Jansen, K., Lahey, R. T., and Akhatoy, I. 2006. "Hydrodynamics Simulation of Air Bubble Implosion Using a Level Set Approach.” Journal of Computer Physics 215: 98-132.

[11] Hara, M., Kaneko, T., and Honda, K. 1987. "Electrical Breakdown Mechanism of Liquid Nitrogen in the Presence of Thermally Induced Bubbles." Journal of Cryogenics 27: 93-101.

[12] Philipp, A., and Lauterborn, W. 1998. "Cavitation Erosion by Single Laser-Produced Bubbles." Journal of Fluid Mechanisms 361: 75-116. 